\title{
A comparative study on the efficacy of fast-track surgery in the treatment of esophageal cancer patients combined with metabolic syndrome
}

\author{
ZITENG ZHANG $^{1 *}$, HAIJUN LI $^{2 *}$, CHUNZHANG YAN ${ }^{3}$, BAOBIN XU $^{1}$, RONGHANG HU $^{1}$, \\ MING MA ${ }^{1}$, HAIXIANG WEI ${ }^{1}$ and YANHONG MENG ${ }^{4}$
}

\begin{abstract}
Departments of ${ }^{1}$ Thoracic Surgery and ${ }^{2}$ Emergency, Affiliated Hospital of Jining Medical University, Jining, Shandong 272000; ${ }^{3}$ Department of Thoracic Surgery, Wenshang People's Hospital, Wenshang, Shandong 272500; ${ }^{4}$ Department of Ultrasonography, Affiliated Hospital of Jining Medical University, Jining, Shandong 272000, P.R. China
\end{abstract}

Received June 15, 2017; Accepted August 14, 2017

DOI: $10.3892 / \mathrm{ol} .2017 .6759$

\begin{abstract}
The purpose of our study was to evaluate the clinical efficacy of fast-track surgery (FTS) in the treatment of esophageal cancer patients combined with metabolic syndrome. Ninety-four esophageal cancer patients with metabolic syndrome were selected in Affiliated Hospital of Jining Medical University from March, 2016 to February, 2017. Patients were randomly divided into control group and observation group with 47 cases in each group. Patients in observation group were treated with FTS, while patients in control group were treated with traditional method. Intraoperative blood loss, the number of dissected lymph nodes, operation time, postoperative hospital stay, the cost of hospitalization, postoperative readmission rate, and incidence of postoperative complications were compared between the groups. Levels of serum inflammatory cytokines (TNF- $\alpha$ and hs-CRP), fat cell factor chemerin and leptin (LP) were detected by enzyme-linked immunosorbent assay (ELISA) at 1 month after surgery. Levels of serum total cholesterol (TC), triglyceride (TG), low-density lipoprotein cholesterol (LDL-C) and high-density lipoprotein cholesterol (HDL-C) at 1 month after surgery were compared between groups. Levels of hemoglobin $(\mathrm{Hb})$, albumin (Alb), prealbumin (PAB) and transferrin (TRF) at 1 month after surgery were also compared between the two groups. Treatment of cancer quality-of-life questionnaireesophageal cancer (OES-18) module was used to evaluate the symptoms of patients at one month after surgery. It turned out
\end{abstract}

Correspondence to: Dr Yanhong Meng, Department of Ultrasonography, Affiliated Hospital of Jining Medical University, 89 Guhuai Road, Jining, Shandong 272000, P.R. China

E-mail: yanhongm17@163.com

${ }^{*}$ Contributed equally

Key words: fast-track surgery, metabolic syndrome, esophageal cancer that no significant differences in intraoperative blood loss, operation time and the number of dissected lymph nodes were found between groups $(\mathrm{p}>0.05)$. Postoperative hospital stay, the cost of hospitalization, postoperative readmission rate and the incidence of postoperative complications were significantly lower in observation group than in control group $(\mathrm{p}<0.05)$. Levels of TNF- $\alpha$, hs-CRP, chemerin and LP in observation group were significantly lower than those in control group at one month after surgery $(p<0.05)$. Levels of TC, TG and LDL-C were significantly lower and HDL-C level was significantly higher in observation group than in control group at one month after surgery $(\mathrm{p}<0.05)$. Levels of $\mathrm{Hb}$ and Alb were significantly lower and levels of PAB and TRF were significantly higher in observation group than in control group at one month after surgery $(\mathrm{p}<0.05)$. OES-18 score of observation group was significantly better than that of control group at one month after surgery $(\mathrm{p}<0.05)$. As a conclusion, FTS can promote postoperative rehabilitation, shorten hospital stay, reduce economic burden and reduce the rehospitalization rate of esophageal cancer patients. At the same time, FTS can also improve the lipid metabolism, nutritional status and regulate the differentiation of adipocytes, alleviate the low inflammatory response state, which in turn promotes metabolic syndrome.

\section{Introduction}

Esophageal cancer is a common malignant tumor of digestive tract. Although incidences of esophageal cancer are different in different regions and races, the incidence is gradually increased worldwide, especially in China and South Africa, esophageal cancer has become one the serious diseases threatening human health $(1,2)$. Treatments of esophageal cancer include radiotherapy, chemotherapy and surgical treatment, and surgical treatment is the only means to cure for patients with resectable cancer (3). With the changes in people's lifestyles and diet structure, dietary factors, especially eating too fast and consuming more saturated fat have become the main causes of esophageal cancer (4). Bad eating habits can also lead to centripetal obesity and dyslipidemia. Esophageal cancer 
patients are usually combined with metabolic syndrome (MS), which brings risks to the prognosis of patients (5). After discharge, excessive nutritional supplement and eating too much high-fat foods can also lead to the occurrence of MS in esophageal cancer patients. Fast-track surgery (FTS) refers to the various effective measures applied preoperatively, intraoperatively and postoperatively with the expectation of reducing the physiological and psychological stress caused by operation, which in turn reduce complications, shorten hospital stay and accelerate rehabilitation (6). In this study, patients with esophageal cancer combined with MS were subjected to FTS intervention and conventional intervention. Efficacy of the two methods was compared. This study provided scientific basis for the treatment and prevention of esophageal cancer combined with MS.

\section{Materials and methods}

General information. Ninety-four esophageal cancer patients combined with metabolic syndrome were selected in Affiliated Hospital of Jining Medical University from March, 2016 to February, 2017. This study was approved by the Ethics Committee of Affiliated Hospital of Jining Medical University. Signed written informed consents were obtained from the patients and/or guardians. Inclusion criteria: i) patients with esophageal cancer diagnosed by CT or MRI, and patients were combined with MS; ii) all patients received surgical treatment; iii) expected survival $>3$ months; iv) signed informed consent. Exclusion criteria: i) patients with abnormal coagulation; ii) unconscious patients or patients with communication disabilities. Patients were randomly divided into control group and observation group with 47 cases in each group. No significant differences in general information were found between groups ( $\mathrm{p}>0.05$ ) (Table I).

\section{Methods}

Preoperative preparation. Preoperative routine examination was performed for all patients in the two groups. Surgical contraindications were excluded and the best timing of surgery was chosen. Patients fasted $8 \mathrm{~h}$ before surgery. Health education and psychological mediation were performed in observation group to relieve their negative emotions, in addition, training on cough, expectoration and breathing was performed for patients in the observation group.

Surgical treatment. All patients underwent tracheal intubation and intravenous anesthesia. Taking left lateral position, thoracoscopy was performed to explore the tumors in thoracic cavity. Mediastinal pleura on both sides of arch of azygos vein were separated. Arch of azygos vein was clamped with Hemo-lock, and arch of azygos vein was cut using ultrasound knife. Lymph nodes under carina, esophageal lymph nodes, mediastinal lymph nodes and lymph nodes next to nervus laryngeus recurrens were dissected. Thoracic cavity was closed with no bleeding detected. Then patient were changed to supine position, and laparoscopy was performed to explore the metastatic nodules in liver and malignant ascites. Ultrasound knife was used to separate stomach, and lymph nodes next to the left gastric artery and around truncus coeliacus were dissected. An incision $(5 \mathrm{~cm})$ was made in the middle of the upper part of abdomen to pull out the stomach, and tubular
Table I. Comparison of baseline information between two groups.

\begin{tabular}{lcccc}
\hline Items & $\begin{array}{c}\text { Control } \\
\text { group }(\mathrm{n}=47)\end{array}$ & $\begin{array}{c}\text { Observation } \\
\text { group }(\mathrm{n}=47)\end{array}$ & $\mathrm{t} / \chi^{2}$ & P-value \\
\hline $\begin{array}{l}\text { Gender } \\
\text { (male/female) }\end{array}$ & $28 / 19$ & $25 / 22$ & 0.389 & 0.533 \\
Age (years) & $45-76$ & $45-75$ & & \\
Average age (years) & $55.86 \pm 6.43$ & $56.03 \pm 6.56$ & 0.127 & 0.899 \\
MAP (mmHg) & $96.87 \pm 12.35$ & $97.35 \pm 11.52$ & 0.195 & 0.846 \\
Tumor staging, $(\%)$ & & & & \\
$\quad$ Stage I & $16(34.04)$ & $13(27.66)$ & 0.533 & 0.762 \\
Stage II & $22(46.81)$ & $23(48.94)$ & & \\
Stage III & $9(19.15)$ & $11(23.40)$ & & \\
\hline
\end{tabular}

stomach was made using three linear cutter staplers on three greater curvatures of the stomach. At the same time, lymph nodes around the left part of esophagus were dissected. Esophagus was pulled out and tubular stomach was lifted up to the neck. A circle cutter stapler was used for cervical esophagus and tubular stomach anastomosis. After that, a drainage tube was placed in the neck and incisions in the neck and abdomen were closed.

Postoperative treatment. Patients in control group were treated with traditional treatment including routine gastrointestinal decompression and fasting for 7 days and fluid infusion. Patients in observation group were treated with FTS including chewing each bite 50 times with food intake controlled by the patients at 1 day after surgery. After each feeding, patients were asked to get out of bed to carry out activities under guidance. Oral feeding was combined with intravenous nutrition to give nutritional support at 3 days after surgery. Intravenous fluid infusion was stopped if oral feeding worked for patients at 4 days after surgery.

\section{Evaluation index}

The surgical outcomes were compared between groups including intraoperative blood loss, the number of dissected lymph nodes, operation time, postoperative hospital stay, the cost of hospitalization, postoperative readmission rate at 3 months after surgery. The incidence of postoperative complications was also compared between groups including arrhythmia, incision infection, recurrent laryngeal nerve injury, pneumonia and other complications.

Levels of serum inflammatory cytokines [tumor necrosis factor- $\alpha(\mathrm{TNF}-\alpha)$ and hs-CRP] and fat cell factor chemerin and leptin (LP) were detected by enzyme-linked immunosorbent assay (ELISA) at 1 month after surgery using kits provided by Beckman Coulter, Inc. (Brea, CA, USA). Fasting peripheral venous blood $(10 \mathrm{ml})$ was extracted from each patients, and automatic biochemical analyzer was used to detect the levels of serum total cholesterol (TC), triglyceride (TG), low-density lipoprotein cholesterol (LDL-C) and high-density lipoprotein cholesterol (HDL-C) and 4 indicators of lipid metabolism including hemoglobin ( $\mathrm{Hb}$ ), albumin (Alb), prealbumin (PAB) and transferrin (TRF) using ELISA kits provided by (Solarbio, Beijing, China). 
Table II. Comparison of surgical outcomes between groups.

\begin{tabular}{lcccc}
\hline Groups & Cases & $\begin{array}{c}\text { Operation } \\
\text { time }(\mathrm{min})\end{array}$ & $\begin{array}{c}\text { Intraoperative } \\
\text { blood loss }(\mathrm{ml})\end{array}$ & $\begin{array}{c}\text { No. of dissected } \\
\text { lymph nodes }\end{array}$ \\
\hline $\begin{array}{l}\text { Observation } \\
\text { group }\end{array}$ & 47 & $264.73 \pm 8.05$ & $56.43 \pm 11.63$ & $27.23 \pm 1.56$ \\
Control group & 47 & $265.24 \pm 8.37$ & $55.86 \pm 18.78$ & $27.56 \pm 2.47$ \\
t-value & & 0.301 & 0.177 & 0.774 \\
P-value & & 0.764 & 0.860 & 0.441 \\
\hline
\end{tabular}

Treatment of cancer quality-of-life questionnaire-esophageal cancer (OES-18) module was used to evaluate the symptoms of patients at one month after surgery (7). One point refers to 'no', 2 points refer to 'a little bit', 3 points refer to 'considerable' and 4 points refer to 'very'. Four symptoms including dysphagia, feeding disturbance, reflux and pain, and 4 single symptom indicators including acataposis, choking feeling, xerostomia, paraesthesia gustatoria, difficulties in cough and difficulties in speaking were scored. Higher scores indicate more obvious symptoms.

Statistical analysis. Data were processed using SPSS 19.0 (SPSS, Inc., Chicago, IL, USA) software. Measurement data were expressed as mean \pm standard deviation (SD), and processed using t-test; count data were expressed as rates and processed using $\chi^{2}$ test. $\mathrm{p}<0.05$ was considered to be statistically significant.

\section{Results}

No significant differences in intraoperative blood loss, operation time and the number of dissected lymph nodes were found between groups ( $\mathrm{p}>0.05)$ (Table II).

Postoperative hospital stay, the cost of hospitalization, postoperative readmission rate and the incidence of postoperative complications were significantly lower in observation group than in control group $(\mathrm{p}<0.05)$ (Table III).

Incidence of complications such as arrhythmia, incision infection, recurrent laryngeal nerve injury and pneumonia was significantly lower in observation group than in control group $(\mathrm{p}<0.05)$ (Table IV).

Levels of inflammatory factors, chemerin and LP at 1 month after surgery were compared between groups. Levels of TNF- $\alpha$, hs-CRP, chemerin and LP in observation group were significantly lower than those in control group $(\mathrm{p}<0.05)($ Table V).

Levels of serum $\mathrm{Hb}, \mathrm{Alb}, \mathrm{TRF}$ and PAB at 1 month after surgery were compared between groups. Levels of $\mathrm{Hb}$ and Alb were significantly lower and levels of PAB and TRF were significantly higher in observation group than in control group at one month after surgery $(\mathrm{p}<0.05)$ (Table VI).

Comparison of lipid metabolism at 1 month after surgery between groups. Levels of TC, TG and LDL-C were significantly lower and HDL-C level was significantly higher in observation group than in control group at one month after surgery $(\mathrm{p}<0.05)$ (Table VII).

OES-18 scores at 1 month after surgery were compared between two groups. OES-18 score in observation
Table III. Comparison of cost of hospitalization, postoperative readmission rate and the incidence of postoperative complications between groups.

\begin{tabular}{lcccc}
\hline Groups & Cases & $\begin{array}{c}\text { Hospital } \\
\text { stay } \\
\text { (day) }\end{array}$ & $\begin{array}{c}\text { Cost of } \\
\text { hospitalization } \\
\text { (yuan) }\end{array}$ & $\begin{array}{c}\text { Readmission } \\
\text { rate, } \mathrm{n}(\%)\end{array}$ \\
\hline $\begin{array}{l}\text { Observation } \\
\text { group }\end{array}$ & 47 & $7.48 \pm 2.26$ & $26885.38 \pm 1365.16$ & $1(2.13)$ \\
$\begin{array}{l}\text { Control group } \\
\text { t } \chi^{2}\end{array}$ & 47 & $11.52 \pm 4.57$ & $3995.73 \pm 2363.78$ & $8(17.02)$ \\
P-value & & 5.433 & 57.488 & 7.388 \\
\hline
\end{tabular}

group was significantly better than that in control group $(\mathrm{p}<0.05)$ (Table VIII).

\section{Discussion}

Esophageal cancer is the result of the interaction between the internal factors and the external environment. It is generally believed that the risk factors for esophageal cancer include esophageal disease (esophageal hiatus, severe reflux esophagitis and Barrett's esophagus), smoking, drinking, bad eating habits, dietary imbalances, obesity, food (fermented food) and genetic factors (8). Esophageal cancer is often diagnosed in the middle and late stage, so the prognosis is poor, and the 5-year survival rate is very low. However, 5-year survival rate of patients with early stage of esophageal cancer can reach $90 \%$. So it would be of great clinical value to carry out early screening and prognosis of esophageal cancer (9). The combination of esophageal cancer and MS can lead to glycolipid metabolic disorders and chronic inflammation, bringing adverse effect to the prognosis of patients.

At present, the surgery is still the major treatment of esophageal cancer. Surgical treatment mainly includes surgical resection of left thoracic esophageal cancer, endoscopic radical operation for esophageal cancer, esophageal cancer resection through the incision on right chest and the middle of abdomen, right chest opening and separate esophagus + stomach dislocation through laparoscopy, cervical anastomosis and Mekeown surgery. With the advantages of small trauma and quick recovery, endoscopic radical surgery of esophageal cancer is currently increasingly widely used in clinic $(10,11)$. Although the level of surgical treatment has been continually improved, the prognosis of patients with esophageal cancer is still relatively poor, especially for patients combined with other diseases (12). The principle of FTS is to integrate preoperative, intraoperative and postoperative treatments to reduce the stress response caused by surgical treatment, which in turn promotes rehabilitation (13). In this study, no significant differences in intraoperative blood loss, operation time and the number of dissected lymph nodes were found between groups ( $\mathrm{p}>0.05$ ); postoperative hospital stay, the cost of hospitalization, postoperative readmission rate and the incidence of postoperative complications were significantly lower in observation group than in control group $(\mathrm{p}<0.05)$. This is because under the guidance of the principle of FTS, health education and psychological counseling as well as training on breathing 
Table IV. Comparison of incidence of complications between groups (n, \%).

\begin{tabular}{|c|c|c|c|c|c|c|}
\hline Groups & Cases & Arrhythmia & $\begin{array}{l}\text { Incision } \\
\text { infection }\end{array}$ & $\begin{array}{l}\text { Recurrent laryngeal } \\
\text { nerve injury }\end{array}$ & Pneumonia & $\begin{array}{l}\text { Total incidence } \\
\text { of complications }\end{array}$ \\
\hline Observation group & 47 & $2(4.26)$ & $0(0.00)$ & $1(2.13)$ & $1(2.13)$ & $4(8.51)$ \\
\hline Control group & 47 & $5(10.64)$ & $2(4.26)$ & $5(10.64)$ & $5(10.64)$ & $17(36.17)$ \\
\hline$t / \chi^{2}$ & & & & & & 8.830 \\
\hline P-value & & & & & & 0.003 \\
\hline
\end{tabular}

Table V. Comparison of levels of inflammatory factors, chemerin and LP between groups.

\begin{tabular}{lccccc}
\hline Groups & Cases & $\begin{array}{c}\text { TNF- } \alpha \\
(\mathrm{ng} / \mathrm{ml})\end{array}$ & $\begin{array}{c}\text { hs-CRP } \\
(\mathrm{mg} / \mathrm{l})\end{array}$ & $\begin{array}{c}\text { Chemerin } \\
(\mu \mathrm{g} / \mathrm{ml})\end{array}$ & $\begin{array}{c}\mathrm{LP} \\
(\mathrm{ng} / \mathrm{ml})\end{array}$ \\
\hline $\begin{array}{l}\text { Observation } \\
\text { group }\end{array}$ & 47 & $1.43 \pm 0.38$ & $1.52 \pm 0.37$ & $93.25 \pm 7.19$ & $11.72 \pm 1.35$ \\
Control group & 47 & $1.98 \pm 0.56$ & $2.03 \pm 0.43$ & $107.38 \pm 9.46$ & $14.48 \pm 1.48$ \\
$\mathrm{t} / \chi^{2}$ & & 5.572 & 6.163 & 8.153 & 9.446 \\
P-value & & $<0.001$ & $<0.001$ & $<0.001$ & $<0.001$ \\
\hline
\end{tabular}

TNF- $\alpha$, tumor necrosis factor- $\alpha$; LP, leptin .

Table VI. Comparison of serum nutrition indexes between two groups.

\begin{tabular}{lccccc}
\hline Groups & Cases & $\mathrm{Hb}(\mathrm{g} / \mathrm{l})$ & $\mathrm{Alb}(\mathrm{g} / \mathrm{l})$ & $\mathrm{PAB}(\mathrm{g} / \mathrm{l})$ & $\mathrm{TRF}(\mathrm{g} / \mathrm{l})$ \\
\hline $\begin{array}{l}\text { Observation } \\
\text { group }\end{array}$ & 47 & $91.43 \pm 5.38$ & $36.52 \pm 3.59$ & $0.27 \pm 0.09$ & $1.78 \pm 0.34$ \\
$\begin{array}{l}\text { Control } \\
\text { group }\end{array}$ & 47 & $96.58 \pm 5.76$ & $41.41 \pm 3.63$ & $0.18 \pm 0.06$ & $1.37 \pm 0.47$ \\
t-value & & & & & \\
P-value & & 4.480 & 6.566 & 5.704 & 4.846 \\
\hline
\end{tabular}

$\mathrm{Hb}$, hemoglobin; Alb, albumin; PAB, prealbumin; TRF, transferrin.

and cough can help patients effectively prevent postoperative aspiration, reduce patient's negative emotions and improve the coordination, which in turn improve the surgical outcomes. Oral feeding at the early stage after surgery can accelerate the recovery of gastrointestinal function. Results also showed that levels of $\mathrm{Hb}$ and $\mathrm{Alb}$ were significantly lower and levels of PAB and TRF were significantly higher in observation group than in control group at one month after surgery. Those results can be explained by the oral feeding at the early stage after surgery, which can provide early nutrition support to restore the necessary postoperative nutrition, thereby speeding up the recovery of patients and reducing unnecessary additional economic expenses. Under the guidance of the principle of FTS, perioperative stress response caused by psychological factors can be reduced, which in turn reduce immunosuppression and the incidence of various postoperative complications (14).

Microinflammation, centripetal obesity, high cholesterol and high fat status is common for esophageal cancer patients combined with MS (15). TNF- $\alpha$, which is mainly from adipose
Table VII. Comparison of lipid metabolism at 1 month after surgery between groups ( $\mathrm{mmol} / \mathrm{l})$.

\begin{tabular}{|c|c|c|c|c|c|}
\hline Groups & Cases & TG & $\mathrm{TC}$ & LDL-C & HDL-C \\
\hline $\begin{array}{l}\text { Observation } \\
\text { group }\end{array}$ & 47 & $1.93 \pm 1.03$ & $4.92 \pm 1.37$ & $2.06 \pm 1.38$ & $1.32 \pm 0.58$ \\
\hline Control group & 47 & $2.64 \pm 1.26$ & $5.76 \pm 1.46$ & $2.78 \pm 1.25$ & $0.89 \pm 0.43$ \\
\hline t-value & & 2.991 & 2.876 & 2.651 & 4.083 \\
\hline $\mathrm{P}$-value & & 0.004 & 0.005 & 0.009 & $<0.001$ \\
\hline
\end{tabular}

TG, triglyceride; TC, cholesterol; LDL-C, low-density lipoprotein cholesterol; HDL-C, high-density lipoprotein cholesterol.

Table VIII. Comparison of OES-18 scores between the two groups.

\begin{tabular}{lcrrrr}
\hline & $\begin{array}{c}\text { Observation } \\
\text { group } \\
(\mathrm{n}=47)\end{array}$ & $\begin{array}{c}\text { Control } \\
\text { group } \\
(\mathrm{n}=47)\end{array}$ & t-value & P-value \\
Items & $6.24 \pm 2.65$ & $13.56 \pm 2.72$ & 13.215 & $<0.001$ \\
Dysphagia & $3.48 \pm 1.35$ & $6.58 \pm 2.47$ & 7.550 & $<0.001$ \\
Feeding disturbance & $5.47 \pm 2.15$ & $11.64 \pm 3.26$ & 10.832 & $<0.001$ \\
Reflux & $1.35 \pm 0.48$ & $4.68 \pm 1.36$ & 15.829 & $<0.001$ \\
Pain & $1.24 \pm 0.28$ & $2.63 \pm 0.37$ & 20.537 & $<0.001$ \\
Acataposis & $5.38 \pm 2.06$ & $12.38 \pm 3.47$ & 11.892 & $<0.001$ \\
Choking feeling & $3.65 \pm 1.42$ & $9.78 \pm 3.08$ & 12.391 & $<0.001$ \\
Xerostomia & $1.47 \pm 0.68$ & $6.39 \pm 1.14$ & 25.410 & $<0.001$ \\
Paraesthesia gustatoria & $7.21 \pm 2.43$ & $13.52 \pm 3.17$ & 10.830 & $<0.001$ \\
Difficulties in cough & $2.36 \pm 0.78$ & $4.18 \pm 1.04$ & 9.598 & $<0.001$ \\
Difficulties in speaking & $2.36 \pm 01$ & & & \\
\hline
\end{tabular}

OES-18, cancer quality-of-life questionnaire-esophageal cancer.

tissue, is a multi-functional inflammatory cytokines with the function of initiating inflammatory response. Hypertrophic adipocytes can enhance the activity of invertase and increase the level of TNF- $\alpha$ in the body (16). Elevated levels of hs-CRP may predict the occurrence of cardiovascular disease (17). TNF- $\alpha$ and hs-CRP secretion can affect the degree of vascular endothelial injury, and vascular endothelial injury can lead to the phosphorylation of insulin receptor, thereby affecting blood lipid metabolism (18). Chemerin is MS-related adipocytokine discovered in recent years (19). LP is a protein hormone secreted by adipose tissue. Chemerin and LP both play an important role in regulating glycolipid metabolism, balancing energy metabolism, promoting cell growth and development, 
especially in regulating adipocyte differentiation and metabolism (20). In this study, levels of TC, TG and LDL-C were significantly lower and HDL-C level was significantly higher in observation group than in control group at one month after surgery, and levels of TNF- $\alpha$, hs-CRP, chemerin and LP in observation group were significantly lower than those in control group $(\mathrm{p}<0.05)$. This may be because under the guidance of the principle of FTS, the normal postoperative nutritional supply can be ensured and stress response and endothelial cell damage can be reduced, which in turn effectively improve the immune disorders, endocrine and the unstable state of internal environment, thereby reducing the microinflammatory state. At the same time, the expression level of chemerin was reduced, which in turn regulated the differentiation and metabolism of adipocytes. LP level was also reduced and LP resistance was improved, fat synthesis was inhibited and energy metabolism was increased. Endocrine was regulated and the level of free fatty acids was reduced, thereby reducing the body's fat content. Results of this study also showed that OES-18 score was significantly better in observation groups than in control group at 1 month after operation $(\mathrm{p}<0.05)$, which indicated that the symptoms of esophageal cancer were improved under the guidance of the principle of FTS. The lower OES-18 score reflected the higher quality of life.

In conclusion, FTS applied for esophageal cancer patients combined with MS in perioperative period can not only ensure the appropriate nutritional support and relieve MS symptoms, but also can reduce postoperative complications, shorten hospital stay, reduce the economic burden of patients and improve rehabilitation. However, this study is still limited by the small sample size. Further study with greater number of samples is needed to confirm the conclusions in this study.

\section{References}

1. Probst A, Aust D, Märkl B, Anthuber M and Messmann H: Early esophageal cancer in Europe: Endoscopic treatment by endoscopic submucosal dissection. Endoscopy 47: 113-121, 2015.

2. Oyama T: Endoscopic submucosal dissection for superficial esophageal cancer. Gastrointest Endosc 65: AB110-AB110, 2015.

3. van der Sluis PC, Ruurda JP, Verhage RJ, van der Horst S, Haverkamp L, Siersema PD, Borel Rinkes IH, Ten Kate FJ and van Hillegersberg R: Oncologic long-term results of robot-assisted minimally invasive thoraco-laparoscopic esophagectomy with two-field lymphadenectomy for esophageal cancer. Ann Surg Oncol 22 (Suppl 3): S1350-S1356, 2015.

4. Shah MA: Update on metastatic gastric and esophageal cancers. J Clin Oncol 33: 1760-1769, 2015.

5. Bonomini F, Rodella LF and Rezzani R: Metabolic syndrome, aging and involvement of oxidative stress. Aging Dis 6: 109-120, 2015.
6. Bu J, Li N, Huang X, He S, Wen J and Wu X: Feasibility of fasttrack surgery in elderly patients with gastric cancer. J Gastrointest Surg 19: 1391-1398, 2015

7. Forootan M, Tabatabaeefar M, Maghsoodi N, Ardeshiri M, Fatemi $\mathrm{M}$ and Rahimzadeh $\mathrm{H}$ : Adaptation of a quality of life questionnaire for Iranian patients with esophageal cancer. Indian J Palliat Care 20: 123-127, 2014.

8. Xu W, Liu Z, Bao Q and Qian Z: Viruses, other pathogenic microorganisms and esophageal cancer. Gastrointest Tumors 2: $2-13,2015$.

9. Mirinezhad SK, Somi MH, Jangjoo AG, Seyednezhad F, Dastgiri S, Mohammadzadeh M, Naseri AR and Nasiri B: Survival rate and prognostic factors of esophageal cancer in east Azerbaijan province, North-west of Iran. Asian Pac J Cancer Prev 13: 3451-3454, 2012.

10. Kinjo Y, Kurita N, Nakamura F, Okabe H, Tanaka E, Kataoka Y, Itami A, Sakai Y and Fukuhara S: Effectiveness of combined thoracoscopic-laparoscopic esophagectomy: Comparison of postoperative complications and midterm oncological outcomes in patients with esophageal cancer. Surg Endosc 26: 381-390, 2012.

11. Kutup A, Nentwich MF, Bollschweiler E, Bogoevski D, Izbicki JR and Hölscher AH: What should be the gold standard for the surgical component in the treatment of locally advanced esophageal cancer: Transthoracic versus transhiatal esophagectomy. Ann Surg 260: 1016-1022, 2014.

12. Zhang SW, Zheng RS, Zuo TT, Zeng HM, Chen WQ and He J: Mortality and survival analysis of esophageal cancer in China. Zhonghua Zhong Liu Za Zhi 38: 709-715, 2016 (In Chinese).

13. Dumans-Nizard V, Guezennec J, Parquin F, Puyo P, Sage E, Abdat R, Vaillant V, Chapelier A, Dreyfus JF, Fischler M, et al; Foch Thoracic Anesthesiology Group: Feasibility and results of a fast-track protocol in thoracic surgery. Minerva Anestesiol 82: 15-21, 2016

14. Bu J and Wu X: Reply to the letter to the editor regarding manuscript entitled: 'Feasibility of fast-track surgery in gastrectomy for elderly patients with gastric cancer'. J Gastrointest Surg 19: 2294-2295, 2015.

15. Lonardo A, Ballestri S, Marchesini G, Angulo P and Loria P: Nonalcoholic fatty liver disease: A precursor of the metabolic syndrome. Dig Liver Dis 47: 181-190, 2015.

16. Tang L, Okamoto S, Shiuchi T, Toda C, Takagi K, Sato T, Saito K, Yokota S and Minokoshi Y: Sympathetic nerve activity maintains an anti-inflammatory state in adipose tissue in male mice by inhibiting TNF- $\alpha$ gene expression in macrophages. Endocrinology 156: 3680-3694, 2015.

17. Tawfik PH, Hafez SS, Mahmoud NH and Sayed HME: Serum Fetuin A, hs-CRP and homocysteine as biochemical markers of cardiovascular complications in chronic dialysis patients. Acta Med Int 2: 57-64, 2015.

18. Johnson AM, Kurti SP, Smith JR, Rosenkranz SK and Harms CA: Effects of an acute bout of moderate-intensity exercise on postprandial lipemia and airway inflammation. Appl Physiol Nutr Metab 41: 284-291, 2016.

19. Bobbert T, Schwarz F, Fischer-Rosinsky A, Maurer L, Möhlig M, Pfeiffer AF, Mai K and Spranger J: Chemerin and prediction of Diabetes mellitus type 2. Clin Endocrinol (Oxf) 82: 838-843, 2015.

20. Dodd GT, Decherf S, Loh K, Simonds SE, Wiede F, Balland E, Merry TL, Münzberg H, Zhang ZY, Kahn BB, et al: Leptin and insulin act on POMC neurons to promote the browning of white fat. Cell 160: 88-104, 2015. 